should have expected this case to have continued a recurrence at the original site. This process was for a much longer period, and perhaps to have seen all the more rapid after attempted extirpation by it finally point at some location lower down the.neck. pastes and caustic lotions. Consequently the imOther cases, very much like this one in character, pression prevailed that surgical interference merely might be mentioned in proof of the value of this postponed the fatal denouement.

remedy.

One more however, might be related with profit:

This was a case of a lad 9 years of age, who had received a traumatic injury of the inferior jaw, by a fall from his bycycle, and which resulted in an extensive acute periostitis, involving the teeth and jaw, from the location of the second temporary molar of the left side, to the ramus of the jaw on the right side; all of the teeth between these points were! loose; pus exuded from the gums at the necks of the teeth, and I feared extensive necrosis.

The treatment adopted was injections of $\mathrm{H}_{2} \mathrm{O}_{2}$ beneath the gums at all points where pus was found to exude. The condition of the anterior part of the mouth began to improve at once, but opposite the first permanent molar, at the lower margin of the jaw, it was necessary a few days later to open an abscess which was about to point at that location. The injections were kept up for two weeks. All discharge had then ceased, the teeth had become firm, and the patient was discharged.

Dr. Harlan has recently called attention to the use of $\mathrm{H}_{2} \mathrm{O}_{2}$ in purulent conditions affecting the maxillary sinus (Archives of Dentistry, p. 204, May, I885), and I would suggest that it will be found equally valuable in the hands of the surgeon in nearly every variety of suppurative inflammation, especially in periostitis, necrosis and deep-seated abscesses, when there is difficulty in completely evacuating the purtulent matter by the ordinary means.

242 Wabash Avenue, Chicago.

\section{CITRIC ACID IN THE EXTIRPATION OF MALIGNANT TUMORS.}

BY C. M. FENN, M.L., of SAN DIEGO, CAL.

Prior to the revelations of the microscope, mor bid growths, with few exceptions, were looked upon as local manifestations of some blood dyscrasia. They were known to be environed by a zone of in filtration or degeneration, of varying area, and the question of recurrence after an operation was supposed to depend largely upon the extent of the incisions. The desire, however, to preserve sym metry, especially if about the face, and to hasten cicatrisation, frequently induced the surgeon to circumscribe his operative procedures, relying subsequently upon antiseptic applications to sterilize flaps and adjacent tissues. Unfortunately, it was seldom possible thus to reach the extreme boundaries of the disease, hidden as they were to sight, and the materies morbi, with lymphatics and blood-vessels as avenues (?), quickly invaded other tissues and viscera; or the increased supply of nutrient material, in the absence of the central mass, soon precipitated
Cellular pathology, however, has modified some of these theories, while confirming others. It has taught us, I think, that tumors, excepting, perhaps, the essentially cancerous ones, are for the most part of local origin, whether homologous or heterologous as to the tissue from which they arise; that the drier ones are seldom recurrent, and that when metastotosis and recurrence take place, it is through the medium of morbid juices, which invade connective and other loose tissues, and set up foci of disease in other portions of the economy. These later investigations, in my opinion, should encourage the surgeon to attempt at least the radical extirpation of all kinds of tumors, though custom and classification may have placed some of them among the "noli me tansere" class.

While yet a student, some twenty years ago, it was my privilege to assist in the removal of a great many epitheliomatous and other abnormal growths, and the rapidity with which some of them recurred impressed me most forcibly at the time. In many instances plastic operations were successfully undertaken to conceal deformity and promote cicatrisation, but at a later period these only added fuel to the flames. In later years, having adopted the nonsystemic theory, and recalling the well-known antagonism of citric acid to diseased tissue, while comparatively innocuous to healthy cells, I devised a method for the extirpation of doubtful and malignant tumors which I shall be glad to see thoroughly tested. It consists, as will be noted, in an attempt to segregate the degenerated zone before using the scalpel.

The notes of a few cases, of dates sufficiently remote to justify conclusion, will best illustrate the

process:
Col. H. T. C., aged 48 , of undoubtedly cancerous antecedents, his grandfather and father having succumbed to malignant disease of the face, had been appealing to thirty-third triturations for the removal of a suspicious tumor situated upon the left ala of the nose. Notwithstanding the attenuations, the disease had rapidly extended down the side of the nose until a large segment of it rested upon the cheek. Its track, fully an inch in length, and more than six lines in width, resembled the - cicatrix of lupus exedens. When first examined by the writer the tumor, now larger than a filbert, had a sessile attachment, an encrusted summit, and, in addition to an indurated margin, was evidently exceedingly vascular. The elderly gentleman, remembering the fate of his ancestors, whose lives had scarcely been prolonged by the frequent operations to which they had been subjected, was naturally very despondent, and while decidedly opposed to the employment of caustics, he was correspondingly incredulous as to any permanent relief to be obtained from the knife.

At the outset my armamentarium included an old hypodermic syringe and a saturated solution of citric 
acid. The needle was introduced deeply into the base of the growth, and about half a drachm of the solution injected. The entire mass became blanched at first, following which a few drops of the acid, mingled with blood, escaped at the surface. Beyond an insignificant tingling, the suffering was so slight that he voluntarily returned on the following day for a repetition of the operation. At intervals of two and five days the process was repeated, until a large zone had been thoroughly saturated with the solution. The size of the tumor gradually diminished until a nodule as large as a small pea alone remained. After the lapse of about three weeks this nucleus was raised upon a tenaculum and excised. The hæmorrhage, as had been anticipated, was quite profuse, but yielded readily to styptics and compression. During the entire period of manipulation, the local irritation seldom required restraining lotions. More than six years have already passed without any evidence of recurrence, and the colonel feels that he has made a fortunate escape.

A second case occurred in the person of a middleaged matron, who also gave a history of a cancerous diathesis in her family. The tumor was situated between the eyes, and was slowly encroaching upon each of them. Immediate excision could not have been undertaken without leaving a most unsightly deformity. Subcutaneous injections of a similar kind were therefore used, the needle point being directed at times toward the centre of the mass, and, again, outwardly, in various directions. A diminution of the induration was soon perceptible to the touch at the periphery of the zone, and continued until an elliptical incision, having a horizontal diameter of not more than twelve lines, included the entire growth. Three years have now elapsed without a return of the disease.

Still another illustration of the vagaries of the cancer and the possibilities of this treatment may not be out of place here. A lady, of perhaps 40 years, stated that about two years previous to our interview, and without any assignable cause, a hard lump made its appearance, a little to the right of and below the right breast. It had been twice operated upon in a distant city, without removing the indurated mammary and axillary glands! After a brief interval of repose it had again come to the surface, and was now manifesting increased virulence. A fungoid mass, as large as the top of a tea-cup, occupied the original site, and was so painful that large doses or opiates only procured temporary relief. It was evident that the system was so saturated with the cancerous juices as to preclude further surgical interference. An operation promising even a tem. porary respite would have involved excision of the breast and axillary glands and an area of integument at least five inches in diameter. As in the other cases, citric acid was injected into the tumor and adjacent tissues, with the results that pain was mitigated, hamorrhage somewhat restrained, besides other evidences of improvement. It was, of course, impossible to stay the progress of the malady by any course of treatment.

San Diego, Cal., July, I885.

\section{MEDICAL PROGRESS.}

\section{MATERIA MEDICA AND THERAPEUTICS.}

Preservation of Alkaloldal Solutions. - Much has been said and written on this subject of late, and many have been the suggestions. In the British Pharmacopocia most of the alkaloid solutions are ordered to be preserved with rectified spirit in certain proportions, the solution of sulphate of atropia being an exception. There cannot be much objection to the use of this preservative when the medicines are intended for internal use per os; but when required for dropping into the eye, ear, or other delicately-lined part of the body, or for subcutaneous injection, the use of spirit then becomes objectionable, because of the smarting pain produced. Liquor atropia, B. P., is a notable example, as also the hypodermic injection of morphia. In place of spirit we have many useful substitutes, or means of avoiding this particular difficulty: $\mathbf{~}$. The use of neutral solutions of the alkaloids with tartaric, salicylic, and other organic acids; 2 . The addition of phenol, thymol, salicylic acid, boracic acid, and such antiseptic substances in minute quantities; 3. The use of camphor-water, glycerine, etc., as the solvent. With regard to the first method, tartrate of morphia may be found a very useful salt for subcutaneous use, as it is readily soluble in water, keeps fairly well, and causes less irritation than the acetic solution. The same applies equally to atropia solution, but it may be mentioned that each of these does not keep for very long periods without the usual fungous formation. The second method may be objected to because of the powerful nature of the substances named, but there need be no fear, because a very small quantity suffices. The salicylates of the alkaloids are known to keep fairly well in solution, even when exposed to the air, and their effect in such a combination is not impaired. Apomorphia solution may contain a trace of phenol to preserve it. Cocaine solutions are said to be useless after two weeks if not preserved by means of some such preparation as a saturated solution of salicylic acid, and very likely this may account for the failure experienced by some practitioners in obtaining the "local anæsthesia" said to be produced so markedly by this recently much used article of materia medica. The third method may be employed with considerable advantage for solutions of atropia, and doubtless also for eseria, pilocarpia, duboisia and gelsemia, when they or their salts are easily soluble in this solvent. Glycerine dissolves some alkaloidal salts very readily, and when diluted with a little water, to thin the product a little, may serve well for hypodermic injection, the pain produced sometimes by the glycerine, especially when used hypodermically, being greatly mollified by the addition of the water. Another method recently recommended by Dr. Macnaughton Jones for the preservation of these solutions is based upon the supposition that light aids in bringing about these changes. He proposes the use of a glass bottle colored blue or yellow, and provided with a vulcanite air-tight stopper, flanged 